Submitted to Acta Cryst. C

\title{
Octasubstituted biphenylenes: is there a favoured conformation?
}

\author{
Peter J. Steel and David A. McMorran¥ \\ Department of Chemistry, University of Canterbury, Christchurch, New Zealand \\ Correspondence email: peter.steel@canterbury.ac.nz \\ $\$$ Current address: Department of Chemistry, University of Otago, PO Box 56 Dunedin, New Zealand
}

\begin{abstract}
Octakis(pyrazol-1-ylmethyl)biphenylene ethanol solvate, $\mathrm{C}_{44} \mathrm{H}_{40} \mathrm{~N}_{16} \cdot \mathrm{C}_{2} \mathrm{H}_{6} \mathrm{O}$, (1) has two independent centrosymmetric molecules, one of which is hydrogen bonded to the solvate molecule. One adopts an arrangement with three arms up and one down in each benzene ring, whilst the other has a conformation with two adjacent arms on the same side of the ring. In neither case is the expected fully alternating form observed.
\end{abstract}

\section{Comment}

We have long been involved in the synthesis and study of new N-heterocyclic ligands for use in coordination and metallosupramolecular chemistry (Steel, 2005). In particular we have prepared a large library of ligands that contain a central arene core to which are appended various heterocycles via flexible linker units (McMorran \& Steel, 2002; McMorran et al., 2004). In the course of designing new ligands, we have employed the concept of "pre-organization" (Hennrich \& Anslyn, 2002). This relies on the principle that six bulky substituents attached to a benzene ring will tend to arrange themselves on alternating faces of the ring in an ababab fashion ( $\mathrm{a}$ and $\mathrm{b}$ being above and below the plane of the ring, as defined by MacNicol et al., 1985). For example, hexakis(pyrazol-1-ylmethyl)benzene adopts this conformation (Hartshorn \& Steel, 1995). A common extension of this approach is to differentiate the two faces of the ring with differing 1,3,5- and 2,4,6-substituents, as for example in 1,3,5-triethyl-2,4,6-tris(pyrazol-1-ylmethyl)benzene (Hartshorn \& Steel, 1997). We were interested to know whether this design concept could be extended to larger aromatic systems, such as biphenylenes. The X-ray crystal structure of octaethylbiphenylene revealed that this compound adopts an ababbaba conformation in the solid state rather than the fully alternating abababab conformation that was calculated to be the most stable (Taha et al., 2000; Marks et al., 2003). However, we previously prepared the new eight armed ligand octakis(2-pyridylmethylsulfanyl)biphenylene and were encouraged to find that in the solid state it was preorganized into the fully alternating abababab conformation (McMorran \& Steel, 2003). We now report the synthesis of octakis(pyrazol-1-ylmethyl)biphenylene and the X-ray crystal structure of its ethanol solvate (1).

The new ligand octakis(pyrazol-1-ylmethyl)biphenylene was prepared by an eightfold phase-transfer catalysed alkylation of octakis(bromomethyl)biphenylene with pyrazole. It was purified by chromatography followed by recrystallization and was characterized by microanalysis, ${ }^{1}$ H NMR spectroscopy and electrospray mass spectrometry. In order to ascertain the conformation of this compound we sought to determine its X-ray structure: the analysis of (1) reveals that the crystals are an ethanol solvate which has two independent half molecules of the ligand in the asymmetric unit. The two independent 


\section{Acta C preprint}

molecules each lie on crystallographic centres of inversion. Figure 1 shows a perspective view of the two molecules with unique and attached atoms labelled. The ethanol solvate molecule is hydrogen bonded to a pyrazole (N62) nitrogen in one molecule of the ligand (Table 2). The planes of the pyrazole rings are inclined to the plane of the adjacent biphenylene unit at angles that range between 67.9 (2) and 112.8 (2) ${ }^{\circ}$. The duplicated pattern of bond lengths and angles within the biphenylene core (see Table 1) also parallels those observed in other structurally characterized octa-substituted biphenylenes (Hubig et al., 2000; Le Magueres et al., 2001a; Le Magueres et al., 2001b; Lu et al., 2002). These suggest that there is some bond delocalization in such molecules.

The two independent molecules have different arrangements of the arms with respect to the biphenylene plane. In the top molecule in Figure 1 the arms have an aababbab arrangement $(\mathrm{C} 1-\mathrm{C} 4)$, whereas in the bottom molecule the arms have an abbabaab arrangement (C1'-C4'). Thus, in neither case do the arms adopt the fully alternating form which might be expected to be energetically most favourable. Figure 2 shows an overlay of the two independent molecules and serves to show that within each benzene ring two arms have very similar orientations, one shows a significant twisting in the orientation of the pyrazole ring and the fourth arm exists on opposite sides of the central plane.

Since all four octasubstituted biphenylenes have different relative orienatations of the substituents it appears that the answer to the title question is that there is a much lower preference for the fully alternating conformation in these derivatives compared to the benzene analogues. We do not believe that the conformations of the two independent molecules are strongly influenced by crystal packing. The molecule with the unusual aababbab (see discussion below) arrangement is not involved in the hydrogen bond mentioned above. Apart from this H-bond, the shortest intermolecular contact is between N82 and H74 of adjacent molecules related by a centre of inversion. This distance is 2.453 (3) $\AA$, which is not unusually short (Mascal, 1998). In order to gain more insight into the reasons for this we carried out a search of the Cambridge Crystallographic Database [version 5.28 (updates January 2007); Allen, 2002] to survey the conformations of octasubstituted naphthalenes which represent a closer analogy to the biphenylenes. Table 3 lists the conformational arrangements of substituents in octasubstituted biphenylenes and naphthalenes. From this it can be seen that octakis(bromomethyl)naphthalene is the only napthalene derivative which has the fully alternating arrangement. It also shows that in all but two examples each ring has two "a" arms and two "b" arms. Furthermore, in all cases the substituents in adjacent peri positions [analogous to $\mathrm{C} 1$ and $\mathrm{C} 4$ in Figure 2, but conventionally labelled $\mathrm{C} 1 / \mathrm{C} 8$ and $\mathrm{C} 4 / \mathrm{C} 5$ for naphthalenes) are on opposite sides of the central plane. This is a well known effect that reduces syn peri steric effects (Marks et al., 2003). Such an effect is certain to be much less important in the biphenylenes than in the naphthalenes. We believe that an important reason for the scarcity of the fully alternating form in the octasubstituted biphenylenes and naphthalenes is that, unlike the hexasubstituted benzenes, this orientation is not centrosymmetric, a situation that minimizes dipole moments.

\section{Experimental}

Octakis(bromomethyl)biphenylene (120 mg, $0.134 \mathrm{mmol})$, pyrazole ( $83 \mathrm{mg}, 1.22 \mathrm{mmol})$, benzene $(15 \mathrm{ml}), 40 \%$ aqueous $\mathrm{KOH}(3 \mathrm{ml})$ and $40 \%$ aqueous $\mathrm{Bu}_{4} \mathrm{NOH}(2$ drops$)$ were refluxed together for $18 \mathrm{~h}$. After cooling, water (10 ml) and ethyl acetate $(20 \mathrm{ml})$ were added, the layers separated and the aqueous layer washed with ethyl acetate $(2 \times 20 \mathrm{ml})$. The combined organic fractions were washed with brine $(10 \mathrm{ml})$ and dried over $\mathrm{MgSO}_{4}$. The solvents were evaporated to give a brown oil which was purified on a silica gel column (ethyl acetate: petroleum ether (50-70) 1:1). Recrystallization from ethanol:petroleum ether (50-70) gave the product as yellow crystals. Yield $54 \mathrm{mg}(51 \%)$. 


\section{Refinement}

Crystal decay was monitored by the measurement of duplicate reflections and was found to be negligible. The OH hydrogen was located from a difference Fourier synthesis and constrained to that position, with $U_{\text {iso }}(\mathrm{H})=1.5 U_{\text {eq }}(\mathrm{O})$. $\mathrm{CH} \mathrm{H}$ atoms were placed in calculated positions, with $\mathrm{C}-\mathrm{H}$ distances set at $0.95 \AA$, and refined as riding, with $U_{\text {iso }}(\mathrm{H})=1.2 U_{\text {eq }}(\mathrm{C})$. Distinction between $\mathrm{N} 2$ and $\mathrm{C} 5$ atoms within the pyrazole rings was made on the basis of alternative refinements and the fact that the $\mathrm{N} 1-\mathrm{N} 2$ bonds are shorter than the $\mathrm{N} 1-\mathrm{C} 5$ bonds (Table 1 ).

\section{Computing details}

Data collection: Bruker SMART (Bruker, 1997); cell refinement: Bruker SMART (Bruker, 1997); data reduction: Bruker SAINT (Bruker, 1997); program(s) used to solve structure: SHELXS97 (Sheldrick, 1990); program(s) used to refine structure: SHELXL97 (Sheldrick, 1997); molecular graphics: Bruker SHELXTL (Bruker, 1997); software used to prepare material for publication: Bruker SHELXTL (Bruker, 1997).

\section{Figures}

Figure 1. Perspective view of the two independent centrosymmetric molecules. Displacement ellipsoids are drawn at the $50 \%$ probability level. Dotted lines represent $\mathrm{H}$ bonds. $\mathrm{H}$ atoms are omitted except for the solvate $\mathrm{OH}$.

Figure 2. Overlay of the two independent molecules, with selected labels to relate to Figure 1. The darker (blue) single colour atoms represent the figure 1a (unprimed atoms) molecule.

\section{(3pjs)}

\section{Crystal data}

$\mathrm{C}_{44} \mathrm{H}_{40} \mathrm{~N}_{16} \cdot \mathrm{C}_{2} \mathrm{H}_{6} \mathrm{O}$

$M_{r}=838.99$

$\gamma=68.719(5)^{\circ}$

Triclinic, $P$ 工

$V=2066.2(14) \AA^{3}$

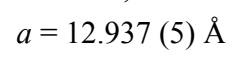

$Z=2$

$b=13.186$ (5) $\AA$

Mo Ka

$c=13.815$ (6) $\AA$

$\mu=0.09 \mathrm{~mm}^{-1}$

$\alpha=87.300(5)^{\circ}$

$T=168(2) \mathrm{K}$

$\beta=70.756(5)^{\circ}$

$0.50 \times 0.50 \times 0.40 \mathrm{~mm}$

\section{Data collection}

Siemens CCD area detector diffractometer

Absorption correction: multi-scan

SADABS (Sheldrick, 2002)

$T_{\min }=0.870, T_{\max }=0.967$

24130 measured reflections
7196 independent reflections

5180 reflections with $I>2 \sigma(I)$

$R_{\text {int }}=0.045$ 


\section{Acta C preprint}

Refinement

$R\left[F^{2}>2 \sigma\left(F^{2}\right)\right]=0.050$

$w R\left(F^{2}\right)=0.126$

$S=1.03$

7196 reflections

569 parameters

Selected geometric parameters $\left(\AA\right.$, $\left.^{\circ}\right)$

$\begin{array}{ll}\mathrm{C} 1-\mathrm{C} 6 & 1.367(3) \\ \mathrm{C} 1-\mathrm{C} 2 & 1.424(3) \\ \mathrm{C} 2-\mathrm{C} 3 & 1.383(3) \\ \mathrm{C} 3-\mathrm{C} 4 & 1.426(3) \\ \mathrm{C} 4-\mathrm{C} 5 & 1.367(3) \\ \mathrm{C} 5-\mathrm{C} 6 & 1.387(3) \\ \mathrm{C} 5-\mathrm{C} 6 & 1 \\ \mathrm{~N} 11-\mathrm{C} 15 & 1.507(3) \\ \mathrm{N} 11-\mathrm{N} 12 & 1.329(3) \\ \mathrm{N} 21-\mathrm{C} 25 & 1.348(2) \\ \mathrm{N} 21-\mathrm{N} 22 & 1.333(3) \\ \mathrm{N} 31-\mathrm{C} 35 & 1.336(3) \\ \mathrm{N} 31-\mathrm{N} 32 & 1.334(3) \\ \mathrm{N} 41-\mathrm{C} 45 & 1.344(3) \\ \mathrm{N} 41-\mathrm{N} 42 & 1.328(3) \\ \mathrm{C} 6-\mathrm{C} 1-\mathrm{C} 2 & 1.343(3) \\ \mathrm{C} 3-\mathrm{C} 2-\mathrm{C} 1 & 115.0(2) \\ \mathrm{C} 2-\mathrm{C} 3-\mathrm{C} 4 & 121.74(19) \\ \mathrm{C} 5-\mathrm{C} 4-\mathrm{C} 3 & 121.86(19) \\ \mathrm{C} 4-\mathrm{C} 5-\mathrm{C} 6 & 114.8(2) \\ \mathrm{C} 4-\mathrm{C} 5-\mathrm{C} 6{ }^{\mathrm{i}} & 123.3(2) \\ \mathrm{C} 6-\mathrm{C} 5-\mathrm{C} 6{ }^{\mathrm{i}} & 146.7(2) \\ \mathrm{C} 1-\mathrm{C} 6-\mathrm{C} 5 & 89.97(17) \\ \mathrm{S}-123.1(2)\end{array}$

$\mathrm{C} 1-\mathrm{C} 6$

$1.367(3)$

$1.383(3)$

$1.387(3)$

$.507(3)$

$333(3)$

$6(3)$

$1.344(3)$

$.328(3)$

$115.0(2)$

$21.74(19)$

$21.86(19)$

Symmetry codes: (i) $-x+2,-y+1,-z$; (ii) $-x+1,-y+2,-z+1$.

Hydrogen-bond geometry $\left(A,{ }^{\circ}\right)$

$D-\mathrm{H} \cdots A$

$D-\mathrm{H}$

0.84

$\mathrm{H} \cdots A$

2.02

$D \cdots A$

$2.828(3)$

$D-\mathrm{H} \cdots A$

O90- $\mathrm{H} 90 \mathrm{~A} \cdots \mathrm{N} 62$

Conformational arrangements of substituents in octasubstituted biphenylenes and naphthalenes

Substituent

Ethyl $^{a}$

2-Pyridylsulfanylmethyl ${ }^{b}$
CSD Refcode

Biphenylenes

CEVDAX

UKOZEZ
Conformation $\left(M^{c}\right.$ Nicol et al., 1995)

ababbaba

abababab
$1.363(3)$

$1.415(3)$

$1.386(3)$

$1.425(3)$

$1.355(3)$

$1.394(3)$

$1.499(3)$

1.339 (3)

1.340 (3)

1.322 (3)

1.348 (3)

1.338 (2)

$1.341(3)$

1.332 (3)

1.339 (3)

115.0 (2)

121.44 (19)

122.03 (19)

114.9 (2)

122.8 (2)

147.1 (2)

90.11 (17)

$123.5(2)$ 
Pyrazol-1-ylmethyl

Phenylsulfanyl (Yellow form) ${ }^{c}$

Phenylsulfanyl (Red form) $)^{\mathrm{c}, \mathrm{d}}$

3-Methylphenylsulfanyl ${ }^{e}$

4-Methylphenylsulfanyl ${ }^{e}$

4-(2-Phenylprop-2-yl)phenylsulfanyl $f^{f}$

3,5-Dimethylphenylsulfanyl ${ }^{g}$

3,4-Dimethylphenylsulfanyl ${ }^{h}$

Cyclohexylsulfanyl $^{\mathrm{i}}$

Phenylselanyl ${ }^{j}$

3-Methylphenoxy ${ }^{k}$

2-Naphthoxy ${ }^{k}$

Bromomethyl

3,3-Dimethylbut-1-enyl ${ }^{m}$ this work

this work

Naphthalenes

BOWWOZ

BOWWOZ01

DEFCAS

DEFCIA

FAJDEZ

TELXEN

TODMAA

KOLXAK

JOTHIJ

JEFDAZ

JEFCUS

WUTRAE

FEWHIZ aababbab

abbabaab

aabbaabb

aabbaabb

aabbaabb

abbabaab

aaabaaab

abababba

aabbaabb

abbabaab

abaababb

abbabaab

ababbaba

abababab

abbabaab

Notes: (a) Taha et al. (2000); (b) McMorran \& Steel (2003); (c) Barbour et al. (1983); (d) Suenaga et al. (2003); (e) MacNicol et al. (1985); ( $f$ ) Downing et al. (1998); ( $g$ ) Downing et al. (1996a); (h) Downing et al. (1996b); (i) MacNicol et al. (1991); (j) MacNicol et al. (1992); (k) Freer et al. (1989); (l) Simaan et al. (2003); (m) Stulgies et al. (2005).

\section{Acknowledgements}

We thank the Royal Society of New Zealand Marsden Fund and James Cook Research Fellowship for funding.

\section{References}

Allen, F. H. (2002). Acta Cryst. B58, 380-388.

Barbour, R. H., Freer, A. A. \& MacNicol, D. D. (1983). Chem. Comm. 362-363.

Bruker (1997). SMART, SAINT and SHELXTL. Area Detector Control and Integration Software. Siemens Analytical X-ray Instruments Inc., Madison, Wisconsin, USA.

Downing, G. A., Frampton, C. S., Gall, J. H. \& MacNicol, D. D. (1996b). Angew. Chem. Int. Ed. Engl. 35, 1547-1549.

Downing, G. A., Frampton, C. S. \& MacNicol, D. D. (1998). Chem. Comm. 2085-2086.

Downing, G. A., MacNicol, D. D., Mallinson, P. R. \& Milligan, P. K. (1996a). Acta Cryst. C52, 2887-2889.

Freer, A. A., MacNicol, D. D., Mallinson, P. R. \& Robertson, C. D. (1989). Tetrahedron Lett. 30, 5787-5790.

Hartshorn, C. M. \& Steel, P. J. (1995). Aust. J. Chem. 48, 1587-1599.

Hartshorn, C. M. \& Steel, P. J. (1997). Chem. Commun. 541-542.

Hennrich, G. \& Anslyn, E. V. (2002). Chem. Eur. J. 8, 2218-2224.

Hubig, S. M., Lindeman, S. V. \& Kochi, J. K. (2000). Coord. Chem. Rev. 200, 831-873.

Le Magueres, P., Lindeman, S. V. \& Kochi, J. K. (2001a). Organometallics, 20, 115-125.

Le Magueres, P., Lindeman, S. V. \& Kochi, J. K. (2001b). J. Chem. Soc., Perkin Trans. 2, 1180-1185.

Lu, J., Zhang, J., Shen, X., Ho, D. M. \& Pascal, R. A. (2002). J. Am. Chem. Soc. 124, 8035-8041.

MacNicol, D. D., Mallinson, P. R. \& Robertson, C. D. (1985). J. Chem. Soc. Chem. Commun. 1649-1651.

MacNicol, D. D., Mallinson, P. R. \& Robertson, C. D. (1992). Acta Cryst. C48, 1557-1558.

MacNicol, D. D., McGregor, W. M., Mallinson, P. R. \& Robertson, C. D. (1991). J. Chem. Soc., Perkin Trans. 1, 3380-3382. 


\section{Acta C preprint}

Marks, V., Gottlieb, H. E. \& Biali, S. E. (2003). Eur. J. Org. Chem. 1825-1835.

Mascal, M. (1998). Chem. Commun. 303-304.

McMorran, D. A. \& Steel, P. J. (2002). Chem. Commun. 2120-2121.

McMorran, D. A. \& Steel, P. J. (2003). Tetrahedron, 59, 3701-3707.

McMorran, D. A., Hartshorn, C. M. \& Steel, P. J. (2004). Polyhedron, 23, 1055-1061.

Sheldrick, G. M. (1990). Acta Cryst. A46, 467-473.

Sheldrick, G. M. (2002). SADABS. Version 2.03. University of Göttingen, Germany.

Sheldrick, G. M. (1997). SHELXL97. University of Göttingen, Germany.

Simaan, S., Marks, V., Gottlieb, H. E., Stanger, A. \& Baili, S. E. (2003). J. Org. Chem. 68, 637-640.

Steel, P. J. (2005). Acc. Chem. Res. 38, 243-250.

Stulgies, B., Prinz, P., Magull, J., Rauch, K., Meindl, K., Ruhl, S. \& de Meijere, A. (2005). Chem. Eur. J. 11, 308-320.

Suenaga, Y., Ueda, A., Kuroda-Sowa, T., Maekawa, M. \& Munakata, M. (2003). Thermochim. Acta, 400, 87-94.

Taha, M., Marks, V., Gottlieb, H. E. \& Baili, S. E. (2000). J. Org. Chem. 65, 8621-8628. 